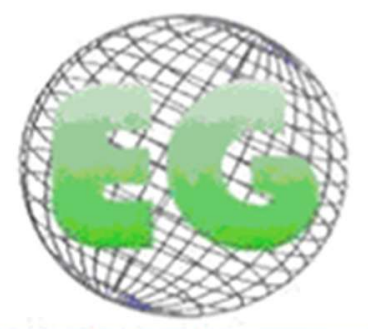

ISSN 1695-6141 $\mathbf{N}^{\circ} 63$

\title{
ORIGINALES
}

\section{Resiliencia asociada a factores de salud mental y sociodemográficos en enfermeros mexicanos durante COVID-19}

Resilience associated to Mental Health and Sociodemographic factors in mexican nurses during COVID-19

\section{Reyna Jazmín Martínez Arriaga ${ }^{1}$ \\ Leivy Patricia González Ramírez ${ }^{2}$ \\ Nancy Evelyn Navarro Ruiz ${ }^{3}$ \\ José María de la Roca Chiapas ${ }^{4}$ \\ Oscar Ulises Reynoso González ${ }^{5}$}

${ }^{1}$ Departamento de Psicología Básica, Centro Universitario de Ciencias de la Salud, Universidad de Guadalajara, México.

${ }^{2}$ Instituto Tecnológico de Monterrey, Escuela de Medicina y Ciencias de la Salud, México.

3 Departamento de Salud Pública, Centro Universitario de Ciencias de la Salud, Universidad de Guadalajara, México.

${ }^{4}$ Departamento de Psicología, Universidad de Guanajuato, Guanajuato, México. josema delaroca@yahoo.com.mx

${ }^{5}$ Centro Universitario de los Altos, Universidad de Guadalajara, México.

\section{https://doi.org/10.6018/eglobal.452781}

Recibido: 26/10/2020

Aceptado: $27 / 03 / 2021$

\section{RESUMEN:}

Introducción: El personal de enfermería es uno de los grupos más afectados por la actual pandemia por COVID-19. Se han reportado problemas de salud mental en esta población, sin embargo, también es importante estudiar la resiliencia, para identificar sus fortalezas. El objetivo de este estudio fue estudiar la resiliencia en enfermeros mexicanos y los factores sociodemográficos y de salud mental asociados a ella. Se llevó a cabo un estudio transversal.

Método: Se envió vía online un cuestionario sociodemográfico y relacionado a COVID-19, así como el Inventario de Resiliencia y el Cuestionario General de Salud-28. Se incluyeron 556 enfermeros, la mayoría fueron mujeres $(80 \%)$, solteras $(60.8 \%)$ y con edades entre $26-35$ años $(38.3 \%)$.

Resultados: Se encontraron niveles bajos de resiliencia en los enfermeros más jóvenes $(p<0.001$, $\left.\eta^{2}=0.05\right)$, solteros $\left(p<0.001, \eta^{2}=0.02\right)$ y con menor nivel educativo $\left(p=0.001, \eta^{2}=0.02\right)$. Los predictores de resiliencia fueron la búsqueda de información sobre salud mental $(\beta=-0.152, p<0.001)$, nivel educativo más alto $(\beta=0.142, p<0.001)$, niveles bajos de depresión $(\beta=-0.307, p<0.001)$ y bajos niveles de disfunción social $(\beta=-0.261, p<0.001)$.

Conclusión: Estos hallazgos permiten identificar los factores asociados a la resiliencia en los enfermeros y cómo estos juegan un rol muy importante en su salud mental. Asimismo, estos datos permiten la identificación de grupos con mayor riesgo psicosocial, con la finalidad de guiar estrategias en salud mental orientadas a aumentar la resiliencia.

Palabras clave: COVID-19, Salud mental, Enfermeros, Enfermería, Resiliencia 


\begin{abstract}
:
Introduction: Nursing personnel are one of the groups which have been most affected by the current COVID-19 pandemic. Although mental health problems have been reported in this population, it is important to study resilience, in order to identify its strengths. The purpose was to study resilience in Mexican nurses and the mental health and sociodemographic factors associated with it. A crosssectional study was used.

Method: A sociodemographic and COVID-19 related questionnaire, the Resilience Inventory and the General Health Questionnaire-28, was sent via online. 556 nurses were included, the majority were women $(80 \%)$, single $(60.8 \%)$, aged between $26-35$ years $(38.3 \%)$.

Results: Lower resilience was found among nurses who were younger $\left(p<0.001, \eta^{2}=0.05\right)$, single $\left(p<0.001, \eta^{2}=0.02\right)$ and with lower levels of education $\left(p=0.001, \eta^{2}=0.02\right)$. Predictors of resilience included the search for mental health information $(\beta=-0.152, p<0.001)$, higher education $(\beta=0.142$, $p<0.001)$, low levels of depression $(\beta=-0.307, p<0.001)$ and low levels of social dysfunction $(\beta=-0.261$, $\mathrm{p}<0.001)$.

Conclusion: This findings allowed to identify the factors which are associated with resilience among nurses and how this plays an important role in their mental. Likewise, this data allows for the identification of high psychosocial risk groups, to better guide mental health strategies aimed at increasing resilience.
\end{abstract}

Key words: COVID-19, Mental health, Nurses, Nursing, Resilience.

\title{
INTRODUCCIÓN
}

La enfermedad por Coronavirus (COVID-19) comenzó a finales del año 2019, y rápidamente se esparció a otras regiones del mundo entero ${ }^{(1)}$. Ante esta situación, el personal de salud es uno de los grupos más vulnerables a padecer problemas de salud mental. Se ha identificado que los enfermeros son quienes han padececido mayores afectaciones psicológicas, tales como ansiedad, depresión y estrés ${ }^{(2)}$. En un estudio realizado por Lai et al (3), encontraron que las enfermeras mujeres que se encontraban en la primera línea de atención, experimentaban síntomas más severos de depresión, ansiedad, insomnio y distress, en comparación con los médicos.

Algunos de los retos que han enfrentado los enfermeros que se encuentran en la primera línea de atención a pacientes infectados son el alto riesgo de contagio por la exposición al virus, no contar con la protección necesaria para prevenir el contagio, la alta carga laboral e incluso el descuido de las actividades básicas mientras se está atendiendo a los pacientes infectados ${ }^{(4)}$. En el estudio reportado por Labrague y de los Santos ${ }^{(5)}$, encontraron que $90 \%$ de los enfermeros refirieron no sentirse totalmente preparados para atender a pacientes con COVID-19. Todo esto ha ocasionado alteraciones en su salud mental, ocasionando altos niveles de estrés y ansiedad, así como falta de apetito, fatiga, dificultad para dormir, llanto excesivo e incluso pensamientos suicidas ${ }^{(6)}$. Sin embargo, se han identificado algunos factores protectores en los enfermeros, que los ha ayudado a enfrentar la actual pandemia. Algunos de ellos son su alto compromiso laboral por la responsabilidad moral y social, una actitud positiva y altos niveles de resiliencia ${ }^{(7)}$. Shechtera, et al. ${ }^{(2)}$, encontraron que algunas estrategias utilizadas por el personal de salud, para afrontar la situación por COVID-19, son la actividad física, la religión, el yoga y la meditación. Se han reportado también niveles moderados de resiliencia en enfermeros que están en la primera línea de atención por COVID-19, asociandose con menores niveles de ansiedad ${ }^{(5)}$.

Estos factores protectores, facilitan una mayor adaptación ante situaciones estresantes y representan un indicador de una adecuada salud mental ${ }^{(8)}$. 


\section{Resiliencia en enfermeros}

El concepto de resiliencia, ha sufrido diversos cambios en los últimos años. Estudios recientes han encontrado que, si bien algunas características personales están asociadas con la resiliencia, ésta es un proceso dinámico, que puede ser modificable y que está determinada por múltiples factores ${ }^{(9)}$.

Según la American Psychological Association (APA), la resiliencia se define como: "el proceso de adaptación adecuada ante la adversidad, trauma, tragedia, amenaza o un estrés significativo" (10). En este sentido, la APA considera que los comportamientos, pensamientos y conductas pueden aprenderse, ya que al no ser considerados como un rasgo de personalidad, la resiliencia es una habilidad que puede ser aprendida (10). Además, se ha encontrado que la resiliencia es un factor predictor para una buena salud mental, física y social (11).

Algunos de los atributos de la resiliencia, que se han identificado en la literatura, incluyen la autoeficacia, optimismo, sentido del humor (8), espiritualidad, actitud positiva, perseverancia y actitud hacia la meta ${ }^{(12)}$.

La resiliencia en enfermeros ha sido ampliamente estudiada, debido a los estresores a los que son sometidos en su práctica cotidiana. Algunos de los factores que provocan altos niveles de estrés en enfermeros, son aspectos organizacionales, el cuidado del paciente, el rol organizacional y dificultades relacionadas con compañeros (13). En una revisión realizada por Badu et al. (11), encontraron una diversidad de atributos individuales que usan los enfermeros para fomentar la resiliencia en sus lugares de trabajo, incluyendo estrategias de mindfulness y de autoeficacia. Argumentan que la autoeficacia tiene que ver con la autosuficiencia, pensamiento positivo, inteligencia emocional, así como pasión e interes por su profesión.

Por su parte, en la revisión realizada por Cooper et al. ${ }^{(8)}$, encontraron que algunos de los atributos de la resiliencia en enfermeros son el apoyo social, la autoeficacia, humor, optimismo y ser realista.

Por otro lado, se han reportado algunas diferencias en los niveles de resiliencia según características sociodemográficas. Se ha encontrado una mayor resiliencia en enfermeros de mayor edad, mayor nivel educativo y aquellos que estaban casados (14). En relación al sexo, se ha reportado que las enfermeras mujeres, llevan a cabo mayores estrategias de afrontamiento para reducir su estrés, en comparación con sus compañeros hombres ${ }^{(7)}$.

Respecto a la relación de la resiliencia con la salud mental de los enfermeros, de acuerdo con Foster et al. (15), es importante estudiar la salud mental en enfermeros en diferentes culturas, con la finalidad de encontrar los factores culturales y ambientales que influyen en la resiliencia.

\section{OBJETIVO}

El objetivo del presente estudio fue evaluar la resiliencia en enfermeros mexicanos, asociada a la salud mental y características sociodemográficas, durante la fase 2 y 3 de la pandemia por COVID-19 en México. 


\section{METODO}

\section{Diseño del estudio y participantes}

Se llevó a cabo un estudio transversal. Se realizó un formulario virtual, a través de un formulario de Google, que fue enviado por ese mismo medio a los participantes. Los participantes fueron sujetos voluntarios, que otorgaron su consentimiento para contestar las preguntas. Se realizó la invitación a través de diversos colegios de enfermeros y universidades en México, y posteriormente se solicitó compartir la encuesta a otros enfermeros para un muestreo por bola de nieve. La encuesta estuvo disponible del 14 de abril al 15 de junio de 2020. Los criterios de inclusión fueron enfermeros o estudiantes en enfermería, que fueran mayores de edad, que radicaran en México y que otorgaran su consentimiento para contestar el formulario.

\section{Mediciones}

Se diseñó un cuestionario para conocer las características sociodemográficas, laborales y aspectos relacionados a COVID-19. Los datos sociodemográficos incluyeron: edad, sexo, estado civil, escolaridad y el estado de residencia. Los datos laborales fueron: el tipo de trabajo (hospitalario o no hospitalario) y horas de trabajo semanales. Por su parte, en las preguntas relacionadas a COVID-19, se preguntó sobre el contacto con pacientes con COVID-19, sobre la atención psicológica recibida a partir de la pandemia por COVID-19 y sobre la búsqueda de información sobre el cuidado de su salud mental. Las preguntas fueron: "Actualmente, ¿tienes contacto con pacientes con COVID-19?" "A partir de la situación por COVID-19, ¿Has recibido atención psicológica?" "A partir de la situación por COVID-19 ¿Has buscado en internet u otras fuentes algún tipo de información o estrategias para cuidar tu salud mental?".

La resiliencia fue evaluada con el Inventario de Resiliencia (IRES), creado y validado en población mexicana, por Gaxiola Romero et al. (12). Este instrumento está conformado por 16 ítems, con 5 opciones de respuesta que va desde "nada" hasta "totalmente". Contempla 7 atributos que caracterizan a las personas resilientes: actitud positiva, sentido del humor, perseverancia, religiosidad, autoeficacia, optimismo y orientación hacia la meta. Se obtuvo una confiabilidad del instrumento de $\alpha=0.93$.

La salud mental fue evaluada con el Cuestionario General de Salud de 28 ítems (GHQ-28, por sus siglas en inglés). Este cuestionario fue desarrollado por Goldberg y Hillier (16), y está basado en la versión original de 60 ítems. Fue validado en población mexicana por Galindo Vázquez et al. (17). EI GHQ-28 contempla 4 sub escalas, de 7 ítems cada una: síntomas somáticos, ansiedad e insomnio, disfunción social y depresión. El $\alpha$ de Cronbach de cada sub escala han obtenido niveles adecuados en población mexicana: síntomas somáticos $\alpha=0.86$, ansiedad y dolor de cabeza $\alpha=0.86$, funcionamiento social $\alpha=0.76$ y depresión e ideación suicida $\alpha=0.80$.

\section{Análisis de datos}

Se realizó estadística descriptiva para todas las variables de estudio. Las variables cuantitativas se expresan con medias (M) y desviaciones estándar (SD). Las variables 
categóricas se expresan en frecuencias y porcentajes. Se realizó la prueba Kolmogorov-Smirnov para evaluar la normalidad de las variables, encontrado una distribución distinta a la normal, por lo que se realizó estadística no paramétrica.

Para las comparaciones de la resiliencia con las variables categóricas, se aplicaron un conjunto de pruebas $\mathrm{U}$ de Mann-Whitney y $\mathrm{H}$ de Kruskal Wallis, dependiendo el número de categorías de cada variable.

Además, se calculó el tamaño del efecto para estimar la magnitud de las diferencias. Particularmente se usó la $r$ de Rosenthal $(r)$ en las comparaciones de dos grupos y la eta cuadrada $\left(\eta^{2}\right)$ en los contrastes de tres o más grupos.

Para las variables cuantitativas, se realizó una correlación bivariada de Rho de Spearman. Posteriormente, se realizó una regresión lineal múltiple, para predecir la variable de resiliencia y sus sub escalas. Se realizaron 8 modelos de regresión lineal: 1) la puntuación total de IRES, 2) actitud positiva, 3) sentido del humor, 4) perseverancia, 5) religiosidad, 6) autoeficacia, 7) optimismo y 8) orientación a la meta. En los modelos sólo fueron consideradas las variables con significancia estadística en las pruebas de comparación y en la correlación. Se utilizó un método "por pasos", para incluir sólo los modelos significativos. Se consideró un nivel de confiabilidad del $95 \%$ y se realizaron las pruebas de colinealidad, así como la verificación de independencia, normalidad y homocedasticidad en los residuales.

\section{Aspectos éticos}

El proyecto fue aprobado por el comité de ética de *** (R-2020-1001-078). Se obtuvo el consentimiento informado de todos los participantes incluidos en el estudio, estableciendo un manejo confidencial de sus datos, así como su aceptación para publicación de resultados.

\section{RESULTADOS}

\section{Características de los participantes}

La muestra estuvo conformada por 556 enfermeros que contestaron el formulario virtual. Los datos socioademográficos, relacionados a COVID-19 y de salud mental, pueden observarse en la tabla 1. El 38.3\% ( $n=213)$ tenían entre 26-36 años de edad, el $80 \%(n=445)$ fueron mujeres, $60.8 \%(n=338)$ solteros, $37.6 \% \quad(n=209)$ licenciados en enfermería. La carga laboral del 36.9\% $(n=205)$ era de 40 horas o más a la semana y el 39.2\% ( $n=226)$ tenía contacto con pacientes COVID-19. La afectación en salud mental más común fue la presencia de "ansiedad-insomnio", en el $21 \%(n=117)$ de los participantes, sin embargo, el 96.6\% $(n=537)$ no había recibido atención psicológica, a pesar de que el 53.8\% ( $n=299)$ había buscado información sobre el cuidado de su salud mental (tabla 1). 
Tabla 1

Resultados descriptivos de las variables de estudio.

\begin{tabular}{|c|c|}
\hline & $\begin{array}{l}N(\%) \\
N=556\end{array}$ \\
\hline \multicolumn{2}{|l|}{ Grupo de edad } \\
\hline$=<25$ años & $211(37.9)$ \\
\hline 26-35 años & $213(38.3)$ \\
\hline$=>36$ años & $132(23.7)$ \\
\hline \multicolumn{2}{|l|}{ Sexo } \\
\hline Femenino & $445(80)$ \\
\hline Masculino & $111(20)$ \\
\hline \multicolumn{2}{|l|}{ Estado civil } \\
\hline Casado/unión libre & $181(32.6)$ \\
\hline Divorciado/separado & $34(6.1)$ \\
\hline Soltero & $338(60.8)$ \\
\hline Viudo & $3(0.3)$ \\
\hline \multicolumn{2}{|l|}{ Escolaridad } \\
\hline Estudiante & $101(18.2)$ \\
\hline Carrera técnica/auxiliar & $114(20.5)$ \\
\hline Lic. En enfermería & 209 (37.6) \\
\hline Especialidad & $65(11.7)$ \\
\hline Posgrado & $67(12.1)$ \\
\hline \multicolumn{2}{|l|}{ Estado de residencia } \\
\hline Noroeste & $19(3.4)$ \\
\hline Noreste & $14(2.5)$ \\
\hline Oeste de México & $363(65.3)$ \\
\hline Este de México & $28(5)$ \\
\hline Centro Norte & $36(6.5)$ \\
\hline Centro Sur & $58(10.4)$ \\
\hline Suroeste & $20(3.6)$ \\
\hline Sureste & $18(3.2)$ \\
\hline \multicolumn{2}{|l|}{ Tipo de trabajo } \\
\hline Hospital /clínico & $226(40.6)$ \\
\hline Otro & $282(50.7)$ \\
\hline \multicolumn{2}{|l|}{ Horas de trabajo } \\
\hline$=<24$ horas & $120(21.6)$ \\
\hline 25-39 horas & $117(21)$ \\
\hline$=>40$ horas & $205(36.9)$ \\
\hline \multicolumn{2}{|c|}{ Contacto con pacientes con COVID-19 } \\
\hline Sí & $218(39.2)$ \\
\hline No & $338(60.8)$ \\
\hline \multicolumn{2}{|c|}{$\begin{array}{l}\text { Atención psicológica recibida a partir de COVID- } \\
19\end{array}$} \\
\hline $\mathrm{Si}$ & $19(3.4)$ \\
\hline No & 537 (96.6) \\
\hline \multicolumn{2}{|c|}{$\begin{array}{l}\text { Búsqueda de información sobre el cuidado de } \\
\text { su salud mental }\end{array}$} \\
\hline $\begin{array}{l}\text { su salud mental } \\
\text { Si }\end{array}$ & $299(53.8)$ \\
\hline No & $257(46.2)$ \\
\hline \multicolumn{2}{|l|}{ GHQ-28 } \\
\hline \multicolumn{2}{|l|}{ Somático } \\
\hline Caso & $47(8.5)$ \\
\hline No caso & 509 (91.5) \\
\hline
\end{tabular}




\begin{tabular}{cl} 
Caso & $117(21)$ \\
No caso & $439(79)$ \\
Disfunción social & \\
Caso & $27(4.9)$ \\
No caso & $529(95.1)$ \\
Depresión & \\
Caso & $14(2.5)$ \\
No caso & $542(97.5)$ \\
\hline
\end{tabular}

\section{Comparación de Resiliencia con las variables de estudio}

En la tabla 2 se pueden observar todas las comparaciones de la resiliencia y sus sub escalas con las variables de estudio.

Se encontraron diferencias estadísticamente significativas según los diferentes grupos de edad. El grupo de $\leq 25$ años fue menos resiliente en comparación con quienes tenían de 26-35 años $(Z=-3.501, p<0.001, r=0.17)$ y con quienes tenían 36 años o más $(Z=-5.457, p<0.001, r=0.29)$, por su parte, el grupo de 36 años o más fue más resiliente que el de 26-35 años $(Z=-2.584, p=0.010, r=0.14)$. Respecto a las sub escalas, el grupo de $\leq 25$ años fue menos resiliente en comparación con el grupo de 26-35 años, en la sub escala de actitud positiva $(Z=-3.466, p=0.001, r=0.17)$, sentido del humor $(Z=-2.458, p=0.014, r=0.12)$, perseverancia $(Z=-2.931, p=0.003, r=0.14)$, religiosidad $(Z=-2.650, p=0.008, r=0.13)$ y autoeficacia $(Z=-3.998, p<0.001, r=0.19)$. El grupo de $\leq 25$ años también fue menos resiliente en comparación con el grupo de $\geq 36$ años, en la escala de actitud positiva $(Z=-4.376, p<0.001, r=0.24)$, sentido del humor $(Z=-2.927, p=0.031, r=0.16)$, perseverancia $(Z=-4.393, p<0.001, r=0.24)$, religiosidad $(Z=-4.934, p<0.001, r=0.27)$, autoeficacia $(Z=-5.409, p<0.001, r=0.29)$ y Orientación a la meta $(Z=-2.060, p=0.039, r=0.11)$.

Por su parte, el grupo de 26-35 años fue menos resiliente en comparación con el grupo de $\geq 36$ años, en la sub escala de perseverancia $(Z=-1.984, p=0.047, r=0.11)$, religiosidad $(Z=-2.707, p=0.007, r=0.15)$ y autoeficacia $(Z=-2.007, p<0.045, r=0.11)$.

Respecto al estado civil, quienes estaban solteros fueron menos resilientes en comparación con quienes estaban casados o en unión libre $(Z=-2.516, p=0.012$, $r=0.11)$ y en comparación con quienes estaban separados o divorciados $(Z=-2.168$, $p=0.030, r=0.11)$. En relación a las sub escalas, quienes estaban solteros fueron menos resilientes en comparación con quienes estaban casados o en unión libre, en la sub escala de actitud positiva $(Z=-2.244, p=0.025, r=0.10)$, perseverancia $(Z=-$ $2.119, p=0.034, r=0.09)$, religiosidad $(Z=-2.995, p=0.003, r=0.13)$ y autoeficacia $(Z=-$ 2.521, $p=0.012, r=0.11)$. Quienes estaban solteros también fueron menos resilientes en comparación con divorciados o separados, en las sub escalas de religiosidad ( $Z=-$ 2.266, $p=0.023, r=0.12)$, autoeficacia $(Z=-2.674, p=0.008, r=0.14)$ y optimismo $(Z=-$ 2.118, $\mathrm{p}=0.034, r=0.11)$.

Respecto a la escolaridad, los estudiantes fueron menos resilientes en comparación con los licenciados en enfermería $(Z=-2.511, p=0.012, r=0.14)$, con los especialistas $(Z=-3.727, p<0.000, r=0.29)$ y con quienes tenían un posgrado $(Z=-3.136, p=0.002$, $r=0.24)$. En las sub escalas, los estudiantes fueron menos resilientes en comparación con los de carrera técnica, sólo en la sub escala de autoeficacia $(Z=-2.138, p=0.033$, 
$r=0.15)$. Los estudiantes también fueron menos resilientes en comparación con los licenciados en enfermería, en las sub escalas de actitud positiva $(Z=-2.853, p=0.004$, $r=0.16)$, sentido del humor $(Z=-3.147, p=0.002, r=0.18)$, religiosidad $(Z=-1.979$, $p=0.048, r=0.11)$ y autoeficacia $(Z=-2.510, p=0.012, r=0.14)$. En comparación con los que tenían una especialidad, los estudiantes también fueron menos resilientes en las sub escalas de actitud positiva $(Z=-3.513, p<0.000, r=0.27)$, perseverancia $(Z=-2.990$, $p=0.003, r=0.23)$, religiosidad $(Z=-3.296, p=0.001, r=0.26)$, autoeficacia $(Z=-3.816$, $p<0.000, r=0.09)$ y orientación a la meta $(Z=-2.225, p<0.026, r=0.17)$. Finalmente, los estudiantes fueron también menos resilientes en comparación con quienes tenían posgrado, en las sub escalas de actitud positiva $(Z=-2.614, p=0.009, r=0.20)$, sentido del humor $(Z=-2.260, p=0.024, r=0.17)$, perseverancia $(Z=-2.287, p=0.022, r=0.18)$, religiosidad $(Z=-2.639, p=0.008, r=0.20)$ y autoeficacia $(Z=-3.775, p<0.000, r=0.29)$.

En relación a las horas de trabajo, únicamente se encontró diferencia en la sub escala de sentido del humor $\left(p=0.010, \eta^{2}=0.01\right)$ (tabla 2$)$. Quienes trabajaban $\geq 40$ horas semanales, tenían menos sentido del humor en comparación con quienes trabajaban $\leq 24$ horas semanales $(Z=-2.994, p=0.003, r=0.17)$.

Table 2 Comparación de resiliencia y sus subescalas con las variables del estudio

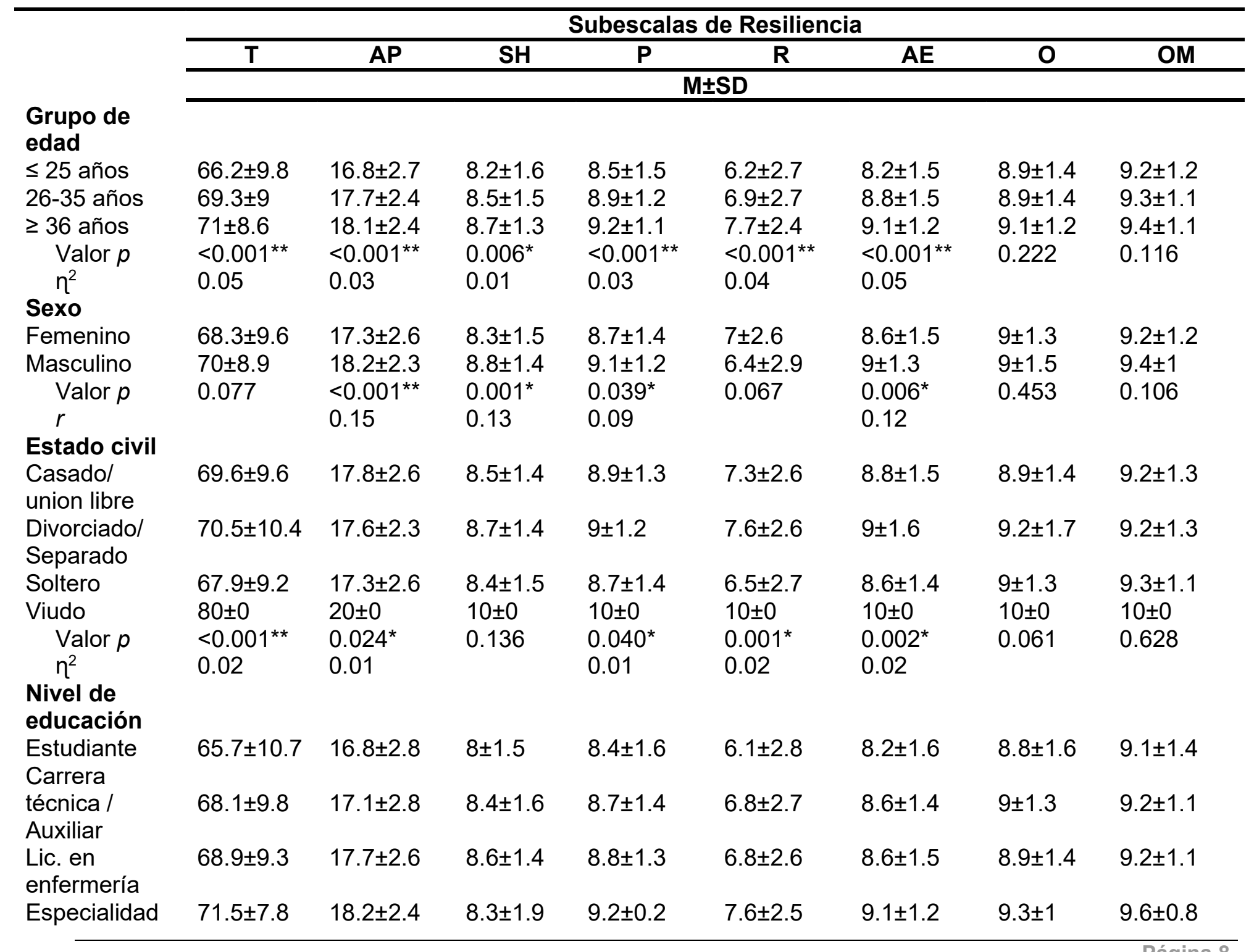




\begin{tabular}{|c|c|c|c|c|c|c|c|c|}
\hline Posgrado & $70.9 \pm 7.4$ & $18 \pm 1.9$ & $8.6 \pm 1.1$ & $9.1 \pm 0.8$ & $7.3 \pm 2.6$ & $9 \pm 1.3$ & $9.1 \pm 1.2$ & $9.5 \pm 0.9$ \\
\hline Valor $p$ & $0.001^{*}$ & $0.001^{*}$ & $0.036^{*}$ & $0.030^{*}$ & $0.008^{*}$ & $<0.001^{* *}$ & 0.420 & 0.053 \\
\hline$\eta^{2}$ & 0.02 & 0.02 & 0.01 & 0.01 & 0.01 & 0.03 & & \\
\hline \multicolumn{9}{|l|}{$\begin{array}{l}\text { Tipo de } \\
\text { trabajo }\end{array}$} \\
\hline Clínico & $69.2 \pm 9$ & $17.6 \pm 2.5$ & $8.5 \pm 1.5$ & $8.8 \pm 1.3$ & $7 \pm 2.6$ & $8.7 \pm 1.4$ & $9 \pm 1.3$ & $9.3 \pm 1.1$ \\
\hline No-clínico & $68.8 \pm 9.7$ & $17.5 \pm 2.6$ & $8.5 \pm 1.5$ & $8.8 \pm 1.4$ & $6.9 \pm 2.7$ & $8.6 \pm 1.5$ & $9 \pm 1.4$ & $9.3 \pm 1.2$ \\
\hline $\begin{array}{l}\text { Valor } p \\
\text { Horas de } \\
\text { trabaio }\end{array}$ & 0.872 & 0.918 & 0.789 & 0.598 & 0.680 & 0.477 & 0.868 & 0.569 \\
\hline$\leq 24$ horas & $69.9 \pm 9.1$ & $17.6 \pm 2.7$ & $8.8 \pm 1.4$ & $8.8 \pm 1.3$ & $7.1 \pm 2.6$ & $8.8 \pm 1.3$ & $9 \pm 1.2$ & $9.3 \pm 1.1$ \\
\hline 25-39 horas & $69.7 \pm 8.9$ & $17.7 \pm 2.4$ & $8.5 \pm 1.4$ & $8.9 \pm 1.3$ & $7.1 \pm 2.7$ & $8.8 \pm 1.3$ & $9.1 \pm 1.3$ & $9.3 \pm$ \\
\hline$\geq 40$ horas & $69.2 \pm 9.2$ & $17.7 \pm 2.4$ & $8.4 \pm 1.5$ & $9 \pm 1.2$ & $7 \pm 2.7$ & $8.7 \pm 1.5$ & $8.9 \pm 1.4$ & $9.3 \pm 1$ \\
\hline Valor $p$ & 0.763 & 0.906 & $0.010^{*}$ & 0.480 & 0.979 & 0.986 & 0.819 & 0.586 \\
\hline \multicolumn{9}{|l|}{$\begin{array}{l}\text { Contacto } \\
\text { con } \\
\text { pacientes } \\
\text { con COVID- } \\
19\end{array}$} \\
\hline Si & $69.1 \pm 9.9$ & $17.6 \pm 2.7$ & $8.5 \pm 1.6$ & $8.9 \pm 1.4$ & $7 \pm 2.8$ & $8.8 \pm 1.4$ & $9 \pm 1.4$ & $9.2 \pm 1$ \\
\hline No & $68.4 \pm 9.2$ & $17.4 \pm 2.5$ & $8.4 \pm 1.4$ & $8.8 \pm 1.3$ & $6.8 \pm 2.6$ & $8.6 \pm 1.5$ & $9 \pm 1.3$ & $9.3 \pm$ \\
\hline Valor $p$ & 0.118 & 0.088 & 0.230 & 0.267 & 0.221 & 0.056 & 0.599 & 0.192 \\
\hline
\end{tabular}

Búsqueda de

información

sobre el

cuidado de

su salud

mental

$\mathrm{Si}$

No

Valor $p$

$69.7 \pm 8.6 \quad 17.8 \pm 2.3$

$8.5 \pm 1.4 \quad 9 \pm 1.2 \quad 7 \pm 2.6$

$8.8 \pm 1.4$

$9 \pm 1.3$

$9.4 \pm 1$

$67.4 \pm 10.2 \quad 17.1 \pm 2.8$

$8.3 \pm 1.6 \quad 8.6 \pm 1.5$

$6.7 \pm 2.8$

$8.5 \pm 1.6$

$8.9 \pm 1.5$

$9.2 \pm 1.3$

$r$

Somático

Caso

$0.11 \quad 0.11$

0.11

0.126

0.146

0.186

0.084

No caso

Valor $p$

$63.9 \pm 11.1 \quad 16.1 \pm 2.7$

$7.8 \pm 1.6 \quad 8 \pm 1.6$

$6.9 \pm 2.7$

$7.8 \pm 1.9$

$8.3 \pm 1.8$

$8.6 \pm 1.6$

$69.1 \pm 9.2 \quad 17.6 \pm 2.6$

$8.5 \pm 1.5$

$8.9 \pm 1.3$

$0.001^{*}$

$<0.001^{\text {** }}$

$6.8 \pm 2.7$

$0.003^{*}$

$<0.001^{\text {** }}$

0.794

$8.7 \pm 1.4$

$9 \pm 1.3$

$0.002^{*}$

$9.3 \pm 1.1$

Ansiedad-

insomnio

Caso

0.14

0.16

0.13

0.13

$<0.001^{* *}$

No caso

Valor $p$

$$
r
$$

$\begin{array}{ll}63.5 \pm 11 & 16 \pm 2.8 \\ 70.1 \pm 8.5 & 17.9 \pm 2.4 \\ <0.001^{* *} & <0.001^{* *} \\ 0.26 & 0.29\end{array}$

$7.8 \pm 1.6$

$8.1 \pm 1.6$

$6.5 \pm 3$

$8.6 \pm 1.4$

$9 \pm 1.2$

$<0.001^{\text {** }}<0.001^{\text {** }}$

$6.9 \pm 2.6$

0.195

7.8 81.7

$8.4 \pm 1.8$

$8.6 \pm 1.6$

$8.9 \pm 1.3$

$<0.001^{\text {** }}$

$9.1 \pm 1.2$

$9.4 \pm 0.9$

$0.21 \quad 0.24$

0.26

0.17

0.25

\section{Disfunción \\ social}

Caso

No caso

Valor $p$

$r$

Depresión

Caso

No caso

Valor $p$

$14.9 \pm 3.1$

$7.2 \pm 1.8$

$7.1 \pm 1.8$

$5.6 \pm 2.7$

$6.9 \pm 2.7$

$<0.001^{* *}$

$<0.001^{* *}$

$8.5 \pm 1.5$

$8.9 \pm 1.3$

$<0.001^{\text {** }}$

$<0.001^{\text {** }}$

$0.013^{*}$

0.16

0.22

0.10

$6.6 \pm 1.9$

$7.5 \pm 2.2$

$7.9 \pm 1.8$

$8.7 \pm 1.4$

$<0.001^{\text {** }}$

$9 \pm 1.3$

$9.3 \pm 1.1$

0.22

0.19

0.24

0.17

$<0.001^{\text {** }}$

$49.6 \pm 12.4 \quad 13.2 \pm 3.2$

$7.2 \pm 1.8 \quad 6.6 \pm 2.2$

$4.3 \pm 2.2$

$5.7 \pm 2$

$6 \pm 2.3$

0.20

$69.2 \pm \quad 17.6 \pm 2.5$

$8.5 \pm 1.5$

$8.9 \pm 1.3$

$6.9 \pm 2.7$

$8.7 \pm 1.4$

$9 \pm 1.2$

$5.4 \pm 2.2$

$<0.001^{\text {** }}<0.001^{\text {** }}$

$0.008^{*}$

$<0.001^{* *}$

$0.001^{*}$

$<0.001^{\text {** }}$

$<0.001^{\text {** }}$

$9.3 \pm 1$

$\begin{array}{lll}0.11 & 0.17 & 0.14\end{array}$

0.21

0.20

$<0.001^{\text {** }}$

0.23 
Abreviaciones: $\mathrm{T}=\mathrm{IRES}$ Total, $\mathrm{AP}=$ Actitud positiva, $\mathrm{SH}=$ Sentido del humor, $\mathrm{P}=$ Perseverancia, $\mathrm{R}=$ Religiosidad, $\mathrm{AE}=$ Autoeficacia, $\mathrm{O}=$ Optimismo, y $\mathrm{OM}$ : Orientación a la meta.

Nota: Las pruebas usadas fueron $U$ de Mann-Whitney y Kruskal Wallis. * $p<0.05,{ }^{*} p<0.001$

\section{Factores asociados a la resiliencia}

En la regresión lineal múltiple se incluyeron todas las variables sociodemográficas, de salud mental y las relacionadas con COVID-19, que fueron significativas de acuerdo a las pruebas $U$ de Mann Whitney, Kruskal Wallis y a la correlación bivariada de Rho de Spearman. Las variables a predecir fueron el puntaje total de resiliencia, así como cada una de sus sub escalas. El modelo final del puntaje total de la resiliencia explica el $28.4 \%$ de la varianza $\left(R^{2}=0.284\right)$ y las variables significativas fueron la depresión $(\beta=-0.307, p<0.001)$, la disfunción social $(\beta=-0.261, \quad p<0.001)$, la búsqueda de información sobre el cuidado de su salud mental $(\beta=-0.152, p<0.001)$ y la escolaridad $(\beta=0.142, p<0.001)$ (tabla 3).

La depresión y la disfunción social fueron los predictores más frecuentes en las subescalas de resiliencia. Ambos fueron predictores de baja actitud positiva ( $\beta=-$ $0.246, p<0.001 ; \beta=-0.152, p=0.001)$, baja perseverancia $(\beta=-0.276, p<0.001 ; \beta=-$ $0.266, p<0.001)$, baja autoeficacia $(\beta=-0.289, p<0.001 ; \beta=-0.255, p<0.001)$, bajo optimismo $(\beta=-0.295, p<0.001 ; \beta=-0.209, p<0.001)$ y baja orientación hacia la meta $(\beta=-0.388, p<0.001 ; \beta=-0.164, p<0.001)$.

Tabla 3. Regresión lineal múltiple sobre las variables asociadas a la resiliência

\begin{tabular}{|c|c|c|c|c|}
\hline & $\begin{array}{c}\mathbf{R}^{2} \\
\text { Ajustada } \\
\end{array}$ & $\bar{\beta}$ & $95 \% \mathrm{Cl}$ & $\bar{p}$ \\
\hline \multicolumn{5}{|l|}{ Total IRES† } \\
\hline GHQ-depresión $\ddagger$ & & -0.307 & -2.657 to -1.531 & $<0.001^{* *}$ \\
\hline GHQ-Disfunción social & & -0.261 & -1.797 to -0.932 & $<0.001^{* *}$ \\
\hline $\begin{array}{l}\text { Búsqueda de } \\
\text { información sobre su } \\
\text { salud mental }\end{array}$ & 0.284 & -0.152 & $\begin{array}{l}-4.238 .033 \text { to }- \\
1.537\end{array}$ & $<0.001^{* *}$ \\
\hline Escolaridad & & 0.142 & 0.558 to 1.661 & $<0.001^{* *}$ \\
\hline \multicolumn{5}{|l|}{ Actitud postitiva } \\
\hline GHQ-Depresión & & -0.246 & -0.631 to -0.303 & $<0.001^{* *}$ \\
\hline GHQ-Disfunción social & & -0.152 & -0.358 to -0.086 & $0.001^{*}$ \\
\hline $\begin{array}{l}\text { Búsqueda información } \\
\text { sobre su salud mental }\end{array}$ & 0.243 & -0.178 & -1.337 to -0.553 & $<0.001^{* *}$ \\
\hline Escolaridad & & 0.131 & 0.125 to 0.441 & $<0.001^{* *}$ \\
\hline $\begin{array}{l}\text { GHQ-ansiedad e } \\
\text { insomnio }\end{array}$ & & -0.134 & -0.230 to -0.046 & $0.003^{*}$ \\
\hline Sexo & & -0.100 & -1.149 to -0.179 & $0.007^{*}$ \\
\hline \multicolumn{5}{|l|}{ Sentido del humor } \\
\hline GHQ-Disfunción social & & -0.218 & -0.281 to -0.095 & $<0.001^{* *}$ \\
\hline $\begin{array}{l}\text { GHQ-Ansiedad } \\
\text { insomnio }\end{array}$ & & -0.190 & -0.174 to -0.047 & $0.001^{*}$ \\
\hline $\begin{array}{l}\text { Búsqueda de } \\
\text { información sobre su } \\
\text { salud mental }\end{array}$ & 0.140 & -0.133 & -0.660 to -0.135 & $0.003^{*}$ \\
\hline $\begin{array}{l}\text { Horas de trabajo } \\
\text { semanales }\end{array}$ & & -0.098 & -0.016 to -0.001 & $0.028^{*}$ \\
\hline
\end{tabular}




\begin{tabular}{|c|c|c|c|c|}
\hline \multicolumn{5}{|l|}{ Perseverancia } \\
\hline GHQ-depresión & & -0.276 & -0.361 to -0.192 & $<0.001^{* *}$ \\
\hline GHQ-Disfunción social & & -0.266 & -0.268 to -0.139 & $<0.001^{* *}$ \\
\hline $\begin{array}{l}\text { Búsqueda de } \\
\text { información sobre su } \\
\text { salud mental }\end{array}$ & 0.258 & -0.156 & -0.637 to -0.233 & $<0.001^{* *}$ \\
\hline Escolaridad & & 0.129 & 0.065 to 0.229 & $<0.001^{* *}$ \\
\hline \multicolumn{5}{|l|}{ Religiosidad } \\
\hline $\begin{array}{l}\text { Edad } \\
\text { GHQ-Depresión }\end{array}$ & 0.071 & $\begin{array}{l}0.201 \\
-0.155\end{array}$ & $\begin{array}{l}0.034 \text { to } 0.081 \\
-0.468 \text { to }-0.146\end{array}$ & $\begin{array}{l}<0.001^{* *} \\
<0.001^{* *}\end{array}$ \\
\hline \multicolumn{5}{|l|}{ Autoeficacia } \\
\hline GHQ-depresión & & -0.289 & -0.407 to -0.223 & $<0.001^{* *}$ \\
\hline GHQ-Disfunción social & & -0.255 & -0.284 to -0.143 & $<0.001^{* *}$ \\
\hline Escolaridad & 0.255 & 0.137 & 0.081 to 0.261 & $<0.001^{* *}$ \\
\hline $\begin{array}{l}\text { Búsqueda información } \\
\text { sobre su salud mental }\end{array}$ & & -0.113 & -0.563 to 0.122 & $0.002^{*}$ \\
\hline \multicolumn{5}{|l|}{ Optimismo } \\
\hline GHQ-depresión & & -0.295 & -0.388 to -0.210 & $<0.001^{* *}$ \\
\hline GHQ-Disfunción social & & -0.209 & -0.231 to -0.094 & $<0.001^{* *}$ \\
\hline Estado civil & 190 & 0.094 & 0.027 to 0.257 & $0.016^{*}$ \\
\hline $\begin{array}{l}\text { Búsqueda información } \\
\text { sobre su salud mental }\end{array}$ & 0.190 & -0.086 & -0.458 to -0.031 & $0.025^{*}$ \\
\hline \multicolumn{5}{|l|}{ Orientación a la meta } \\
\hline GHQ-depresión & & -0.388 & -0.403 to -0.259 & $<0.001^{* *}$ \\
\hline GHQ-Disfunción social & & -0.164 & -0.163 to -0.052 & $<0.001^{* *}$ \\
\hline $\begin{array}{l}\text { Búsqueda de } \\
\text { información sobre su } \\
\text { salud mental }\end{array}$ & 0.246 & -0.106 & -0.425 to -0.078 & $0.005^{*}$ \\
\hline
\end{tabular}

Abreviaciones: IRES= Inventario de Resiliencia, GHQ=General Health Questionnaire (Cuestionario General de Salud).

${ }^{*} \mathrm{p}<0.05$

${ }^{*} p<0.001$

\section{DISCUSIÓN}

Ante la situación del COVID-19, el personal de enfermería es el que más se ha visto afectado respecto a su salud mental, debido a las características de su labor (2), por lo que se hace importante investigar la resiliencia en los enfermeros ante la pandemia actual.

En el presente estudio, los enfermeros que presentaban síntomas somáticos, ansiedad-insomnio, disfunción social y depresión, fueron quienes tuvieron menores niveles de resiliencia. La evidencia ha mostrado cómo la resiliencia ayuda a mantener menores niveles de ansiedad y depresión (15) y de síntomas somáticos como dolores de cabeza y cuello, tensión muscular y fatiga ${ }^{(18)}$. De forma similar, en este estudio, la depresión y la disfunción social fueron los predictores más comunes para bajos puntajes de resiliencia y sus atributos: actitud positiva, sentido del humor, perseverancia, autoeficacia, optimismo y orientación a la meta. Este hallazgo fortalece la evidencia sobre el importante rol de la resiliencia en la salud mental de los enfermeros. 
En relación a los atributos de la resiliencia, se encontró que la perseverancia y la autoeficacia de los enfermeros están asociadas con la depresión, disfunción social, la escolaridad y la búsqueda de información sobre salud mental. La perseverancia y la autoeficacia se han descrito como rasgos positivos de personalidad, particularmente en la consecución de una meta, tal como la participación deportiva ${ }^{(19)}$. En el contexto de enfermería, la autoeficacia es encontrada en aquellos enfermeros que tienen un nivel educativo más elevado ${ }^{(14)}$. Por su parte, el optimismo estuvo asociado con la depresión, la disfunción social, el estado civil y la búsqueda de información sobre salud mental. El optimismo, es uno de los atributos de la resiliencia que más se ha ligado con una mejor salud. Kim et al. (20), encontraron una fuerte asociación el optimismo y una disminución de riesgos de mortalidad, incluyendo el cáncer, enfermedades del corazón, enfermedades respiratorias e infecciones. Sin embargo, ellos sugieren que es importante considerar el papel del optimismo en las conductas saludables, y por ende, que estos últimos estén vinculados con la disminución de riesgos. Estos hallazgos sugieren la importancia de la promoción del optimismo como factor protector para la salud.

Respecto a las características sociodemográficas, se encontró también que los enfermeros más jóvenes, solteros y con una escolaridad más baja, presentaron significativamente menores niveles de resiliencia.

En relación a la diferencia por edad, es consistente en la literatura el bajo nivel de resiliencia en enfermeros más jóvenes ${ }^{(13,14)}$. Foster, et al. ${ }^{(13)}$, encontraron que los enfermeros más jóvenes presentaron menos resiliencia, debido a su dificultad para comprender y controlar pensamientos negativos relacionados a eventos adversos. Esta diferencia puede deberse también a los años de experiencia de los enfermeros, ya que se ha encontrado que la resiliencia es más elevada en quien cuenta con mayor experiencia laboral (21). Respecto a los atributos de la resiliencia, a partir de la regresión, se encontró que la edad predecía altos niveles de religiosidad. En estudios previos, se ha reportado que la religiosidad es más común en mujeres de edades más avanzadas, y que ésta puede ser un factor protector para padecimientos psicológicos como la depresión, en este grupo etario (22). Sin embargo, es importante tomar en cuenta las diferencias de cada región, ya que se ha encontrado una mayor prevalencia de prácticas religiosas en algunas regiones de latinoamérica ${ }^{(22)}$.

Respecto al sexo, lo encontrado en la literatura es contradictorio. En el presente estudio, los hombres tuvieron mayor resiliencia, en los atributos de actitud positiva, sentido del humor, perseverancia y autoeficacia; sin embargo, el tamaño del efecto fue relativamente bajo: $r=0.15, r=0.13, r=0.09$ y $r=0.12$, respectivamente. De forma similiar, en diversos estudios han encontrado menores niveles de resiliencia menores en las mujeres en comparación con hombres $(14,23)$. Sin embargo, también se ha reportado en enfermeros, que no hay diferencias significativas en la resiliencia según el sexo ${ }^{(24)}$. Esta contrariedad puede deberse a que la mayoría de los participantes en estudios con enfermeros son mujeres, por lo que podría no haber una representatividad del sexo masculino.

Por otro lado, se encontró que quienes estaban solteros fueron menos resilientes, en comparación con quienes estaba casados o en unión libre y con quienes estaban separados o divorciados. Específicamente, fueron menos resilientes en los atributos de actitud positiva, perseverancia, religiosidad y autoeficacia. Nuestros resultados apoyan los resultados de estudios previos, que reportan mayores niveles de 
resiliencia en personas casadas $(14,25)$. Estar casado o vivir en pareja, puede fungir como un factor protector, debido a que permite estar conectados con otras personas y proveer apoyo para enfrentar situaciones estresantes ${ }^{(14)}$. Ante la pandemia actual, se ha encontrado en población mexicana, que estar soltero está asociado con un mayor estrés psicológico ${ }^{(26)}$.

Por otra parte, quienes tenían un nivel de escolaridad más bajo, fueron quienes tuvieron menores niveles de resiliencia, en este caso los estudiantes y quienes tenían una carrera técnica. Asimismo, la escolaridad fue predictor para la autoeficacia, perseverancia y la actitud positiva. Ya en la literatura se reporta, que los enfermeros con un nivel de escolaridad más alto, presentan mayores niveles de resiliencia ${ }^{(21)}$. De hecho, se han vinculado a los enfermeros que tienen un posgrado, con mayores niveles de autoeficacia ${ }^{(14)}$, similar a los hallazgos de este estudio, que quienes tenían niveles de posgrado o especialidad, fueron quienes tenían una mayor autoeficacia.

Los programas en salud mental dirigidos a enfermeros, para promover la resiliencia, han mostrado eficacia ${ }^{(27)}$. Sin embargo, uno de los obstáculos para la implementación de estos programas es el rechazo por parte de los enfermeros de recibir apoyo psicológico. En este estudio se encontró que más de la mitad de los enfermeros $(53.8 \%)$ había buscado información sobre su salud mental ante la situación de COVID-19, sin embargo, sólo $3.4 \%$ había recibido atención psicológica debido a la pandemia. Si bien en algunas poblaciones, los enfermeros aceptan muy bien las intervenciones psicológicas ${ }^{(11)}$, algunos han referido que no necesitan apoyo en salud mental $(7,28)$. Cai et al. $(7)$, reportaron hasta un $36 \%$ de enfermeros que no consideraban importante el apoyo psicológico. Esto sugiere un reto para la implementación de programas de salud mental en enfermeros, por lo que sugerimos que, además de diseñar e implementar los programas, se generen estrategias para interesar a la población de enfermería. Otra alternativa puede ser, considerar el apoyo de teléfonos móviles, para la implementación de aplicaciones digitales, orientadas a enseñar algunas estrategias psicológicas que aumenten la resiliencia ${ }^{(29)}$.

Algunas estrategias para mejorar la salud mental en enfermeros ante la actual pandemia por COVID-19, son: fomentar el trabajo en armonía con los compañeros de trabajo, promover la expresión emocional ya sea hablando, pintando o cantando, implementar estrategias de relajación, como la respiración profunda o el ejercicio, comunicarse con colegas que tuvieron la misma experiencia o sentimientos similares, mejorar la educación sobre las medidas de prevención ante la exposición de riesgo, con la finalidad de reducir preocupaciones de contagio, establecer una red de apoyo social y buscar apoyo profesional si sienten que el estrés psicológico es elevado y no disminuye ${ }^{(6)}$.

Una de las limitaciones del presente estudio fue la gran participación de mujeres, por lo que se recomienda en futuros estudios, incluir una muestra más heterogénea. Además, se sugiere incluir enfermeros en condiciones laborales diferentes, así como investigar otras variables protectoras de salud mental, como afrontamiento y apoyo social. 


\section{CONCLUSIÓN}

La pandemia actual ha presentado grandes retos en el personal de enfermería a nivel mundial. En este estudio se encontró que los problemas de salud mental como la ansiedad e insomnio, la depresión, la disfunción social y los síntomas somáticos, se asocian con una menor resiliencia. Esto hallazgo sugiere el importante rol que juega la resiliencia como protector para problemas de salud mental. Asimismo, encontramos que quienes tenían más edad, vivían en pareja y tenían una escolaridad más alta, fueron quienes presentaron significativamente mayor resiliencia. Esto permite la identificación de los grupos de riesgo para orientar las estrategias en salud mental en enfermeros. Además, es necesario que existan en los hospitales y en los distintos niveles de atención en salud, departamentos de atención a la salud mental de los trabajadores, en especial los que estén en contacto con pacientes de riesgo de muerte, o alta incidencia de mortalidad.

Asimismo, el presente estudio tiene diversas implicaciones para la atención psicosocial de enfermería ante la pandemia actual. Se sugiere reforzar la atención psicosocial en aquellos enfermeros que tienen problemas de salud mental como ansiedad, depresión, disfunción social y síntomas somáticos, así como en enfermeros jóvenes, solteros y con una escolaridad más baja. Es importante que se dirijan programas en salud mental al personal de enfermería, y que estos incluyan estrategias que fomenten la resiliencia. Algunas de ellas pueden incluir el reconocimiento de emociones, fomentar la espiritualidad y reflexibilidad, la autoeficacia, el optimismo, el humor, la actitud positva, la perseverancia y la actitud hacia la meta.

\section{REFERENCIAS}

1. Dong $M$ \& Zheng J. Letter to the editor: Headline stress disorder caused by Netnews during the outbreak of COVID-19. Health Expect. 2020;23(2): 259-260. Available in: https://doi.org/10.1111/hex.13055

2. Shechter A, Diaz F, Moise N, Anstey E, Ye S, Agarwal S, et al. Psychological distress, coping behaviors, and preferences for support among New York healthcare workers during the COVID-19 pandemic. Gen Hosp Psychiatry. 2020;66:1-8. Available in: https://doi.org/10.1016/i.genhosppsych.2020.06.007

3. Lai J, Ma S, Wang Y, Cai Z, Hu J, Wei N, et al. Factors Associated With Mental Health Outcomes Among Health Care Workers Exposed to Coronavirus Disease 2019. JAMA Netw Open. 2020; 3(3): e203976. Available in: https://doi.org/10.1001/jamanetworkopen.2020.3976

4. Smith GD, Ng F \& Cheung Li WH. COVID-19: Emerging compassion, courage and resilience in the face of misinformation and adversity. J Clin Nurs. 2020;29:14251428. Available in: https://doi.org/10.1111/jocn.15231

5. Labrague L. \& De los Santos JA. COVID-19 anxiety among frontline nurses: predictive role of organisational support, personal resilience and social support. $\mathrm{J}$ Nurs Manag. 2020;00:1-9. Available in: https://doi.org/10.1111/jonm.13121

6. Shen X, Zou X, Zhong X, Yan J \& Li L. Psychological stress of ICU nurses in the time of COVID-19. Crit Care. 2020;24:00. Available in: https://doi.org/10.1186/s13054-020-02926-2 
7. Cai H, Tu B, Ma J, Chen L, Fu L, Jiang Y, et al. Psychological Impact and Coping Strategies of Frontline Medical Staff in Hunan Between January and March 2020 During the Outbreak of Coronavirus Disease 2019 (COVID-19) in Hubei, China. Med Sci Monit. 2020;26:e924171. Available in: https://doi.org/10.12659/MSM.924171

8. Cooper AL, Brown JA, Rees CS \& Leslie GD. Nurse resilience: A concept analysis. Int J Ment Health Nurs. 2020;29:553-575. Available in: https://doi.org/10.1111/inm.12721

9. Chmitorz A, Kunzler A, Helmreich I, Tüscher O, Kalisch R, Kubiak T, et al. Intervention studies to foster resilience - A systematic review and proposal for a resilience framework in future intervention studies. Clin Psychol Rev. 2018;59:78100. Available in: https://doi.org/10.1016/j.cpr.2017.11.002

10. American Psychological Association [Internet]. Washington, DC; 2012 [cited 2020 Aug 7]. Building your resilience: We all face trauma, adversity and other stresses. Here's a roadmap for adapting to life-changing situations, and emerging even stronger than before. Available in: https://www.apa.org/topics/resilience.

11. Badu E, O'Brien AP, Mitchell R, Rubin M, James C, McNeil K, et al. Workplace stress and resilience in the Australian nursing workforce: A comprehensive integrative review. Int $J$ Ment Health Nurs. 2020;29:5-34. Available in: https://doi.org/10.1111/inm.12662

12. Gaxiola Romero JC, Frías Armenta M, Hurtado Abril MF, Salcido Noriega LC \& Figueroa Franco M. Validación del Inventario de Resiliencia (IRES) en una muestra del Noroeste de México. Enseñanza e Investigación en Psicología. 2011;16(1):73-83. Available in: https://www.redalyc.org/pdf/292/29215963006.pdf

13. Foster K, Roche M, Giandinoto JA \& Furness T. Workplace stressors, psychological well-being, resilience, and caring behaviours of mental health nurses: A descriptive correlational study. Int J Ment Health Nurs. 2019;29:56-68. Available in: https://doi.org/10.1111/inm.12610

14. Ang SY, Uthaman T, Ayre TC, Mordiffi SZ, Ang E \& Lopez V. Association between demographics and resilience - a cross-sectional study among nurses in Singapore. Int Nurs Rev. 2018;65:459-466. Available in: https://doi.org/10.1111/inr.12441

15. Foster K, Roche M, Delgado C, Cuzzillo C., Giandinoto, JA \& Furness, T. Resilience and mental health nursing: An integrative review of international literature. Int J Ment Health Nurs. 2019;28(1):71-85. Available in: https://doi.org/10.1111/inm.12548

16. Goldberg DP \& Hillier VF. A scaled version of the general health questionnaire. Psychol Med. 1979;9(1):139-45. Available in: https://doi.org/10.1017/s0033291700021644

17. Galindo Vázquez O, Meneses García A, Herrera Gómez A, Cabrera Galeana P, Suchil Bernal L, Rivera Fong L, et al. Propiedades psicométricas del Cuestionario General de Salud de Goldberg-GHQ-28- en cuidadores primarios informales de pacientes con cáncer. Psicooncología. 2018;14(1):71-81. Available in: https://doi.org/10.5209/PSIC.55812

18. Smith B, Shatté A, Perlman A, Siers M \& Lynch WD. Improvements in Resilience, Stress, and Somatic Symptoms Following Online Resilience Training: A DoseResponse Effect. J Occup Environ Med. 2018;60(1):1-5. Available in: https://doi.org/10.1097/JOM.0000000000001142

19. Laborde S, Guillén F, Dosseville F \& Alle MS. Chronotype, sport participation, and positive personality-trait-like individual differences. Chronobiol Int. 2015;32(7):942951. Available in: https://doi.org/10.3109/07420528.2015.1055755 
20. Kim ES, Hagan KA, Grodstein F, DeMeo DL, De Vivo I \& Kubzansky LD. Optimism and Cause-Specific Mortality: A Prospective Cohort Study. Am J Epidemiol. 2017;185(1):21-29. Available in: https://doi.org/10.1093/aje/kww182

21.Zheng Z, Gangaram P, Xie H, Chua S, Ong SBC \& Koh SE. Job satisfaction and resilience in psychiatric nurses: A study at the Institute of Mental Health, Singapore. International Journal of Mental Health Nursing. 2017;26(6):612-619. Available in: https://doi.org/10.1111/inm.12286

22. Santero M, Daray FM, Prado C, Hernández-Vásquez A \& Irazola V. Association between religiosity and depression varies with age and sex among adults in South America: Evidence from the CESCAS I study. PLoS One. 2019;14(12): e0226622. Available in: https://doi.org/10.1371/journal.pone.0226622

23. Rodriguez-Llanes JM, Vos F \& Guha-Sapir D. Measuring psychological resilience to disasters: are evidence-based indicators an achievable goal? Environ Health. 2013;12:115. Available in: https://doi.org/10.1186/1476-069X-12-115

24. Kutluturka S, Sozeri E, Uysual N \& Bay F. Resilience and burnout status among nurses working in oncology. Ann Gen Psychiatry. 2016;14;15:33. Available in: https://doi.org/10.1186/s12991-016-0121-3

25. Hwang JH \& Yu CS. Depression and resilience in ulcerative colitis and Crohn's disease patients with ostomy. Int Wound J. 2018;1:62-70. Available in: https://doi.org/10.1111/iwj.13076

26. González-Ramírez L, Martínez-Arriaga RJ, Hernández-González MA \& De la Roca-Chiapas JM. Psychological Distress and Signs of Post-Traumatic Stress in Response to the COVID-19 Health Emergency in a Mexican Sample. Psychol Res Behav Manag. 2020;13: 589-597. Available in: https://doi.org/10.2147/PRBM.S259563

27. Henshall C, Davey Z \& Jackson D. The implementation and evaluation of a resilience enhancement programme for nurses working in the forensic setting. Int $\mathrm{J}$ Ment Health Nurs. 2020;29:508-520. Available in: https://doi.org/10.1111/inm.12689

28. Chen Q, Liang M, Li Y, Guo J, Fei D, Wang L, et al. Mental health care for medical staff in China during the COVID-19 outbreak. Lancet Psychiatry. 2020;7(4):e15e16. Available in:https://doi.org/10.1016/S2215-0366(20)30078-X

29. Reyes AT. A Mindfulness Mobile App for Traumatized COVID-19 Healthcare Workers and Recovered Patients: A Response to "The Use of Digital Applications and COVID-19". Community Ment Health J. 2020;56(7):1204-1205. Available in: https://doi.org/10.1007/s10597-020-00690-9

ISSN 1695-6141

(C) COPYRIGHT Servicio de Publicaciones - Universidad de Murcia 